\title{
Association of Next Generation Sequencing and Routine Testing with Survival of Advanced Non- Small Cell Lung Cancer Patients in Clinical Practice
}

\section{Wenxian Wang}

Zhejiang Cancer Hospital

Jinfei Si

Zhejiang Cancer Hospital

Xiaoyan Zhang

Genetron Health (Beijing) Technology, Co. Ltd.

Tonghui Ma

Genetron Health (Beijing) Technology, Co. Ltd.

\section{Guangyuan Lou}

Zhejiang Cancer Hospital

\section{Zhiyong Shi}

Zhejiang Cancer Hospital

\section{Lan Shao}

Zhejiang Cancer Hospital

\section{Yiping Zhang}

Zhejiang Cancer Hospital

Zhengbo Song ( $\nabla$ zheng_bo_song@163.com )

Zhejiang Cancer Hospital

\section{Research}

Keywords: Next generation sequencing, non-small cell lung cancer, EGFR, survival, targeted therapy

Posted Date: September 28th, 2021

DOI: https://doi.org/10.21203/rs.3.rs-917433/v1

License: (c) (i) This work is licensed under a Creative Commons Attribution 4.0 International License. Read Full License 


\section{Abstract}

Background: The rapid development of detection technologies has resulted in an increased use of next generation sequencing (NGS) in advanced non-small cell lung cancer (NSCLC) patients. Here, we investigated the clinical benefits of different detection technologies for patients with advanced lung cancer in a real world setting.

Methods: This retrospective study enrolled advanced NSCLC patients diagnosed between January 1, 2013 and June 30, 2019 in Zhejiang Cancer Hospital who received genetic testing. Patients received either NGS or routine testing (EGFR and/or ALK and/or ROS1 only). Although different panels were used for NGS, they all included nine genes mentioned as molecular biomarkers in the NCCN guidelines (EGFR, ALK, ROS1, KRAS, HER2, BRAF, MET, RET, and NTRK). Clinical outcomes were compared between advanced NSCLC patients who received NGS and those undergoing routine testing. Overall survival (OS) and survival rates were analyzed. Propensity score analysis was used to prevent survival bias associated with patient characteristics.

Results: Of 6,451 patients with advanced NSCLC included in the analysis, 5,666 (87.8\%) patients underwent routine testing and 785 (12.2\%) were tested by NGS. The median OS was 22.6 months and the 5 -year survival rate was $22.89 \%$. Comparison of OS showed an absolute difference between the two cohorts according to unadjusted survival curves (24.8 months vs. 22.1 months, $P=0.0097$ ), whereas no difference in OS was observed using propensity score analysis $(P=0.533)$. The 1-year survival rate was significantly higher in patients tested by NGS than in those undergoing routine testing ( $82.0 \%$ vs. $70.7 \%$, $\mathrm{P}=0.0004)$. In patients with uncommon mutations and in those receiving targeted therapies, propensity score analysis showed that the 1- and 2-year mortalities were significantly lower in the NGS group than in the routine testing group without mutations. This could be due to the higher rate of patients receiving targeted therapy $(68.0 \%$ vs. $53.6 \% ; P=0.042)$ and off-label drugs $(28.10 \%$ vs. $11.40 \% ; P=0.002)$ in the NGS testing cohort.

Conclusions: Although propensity score analysis showed NGS had no significant benefit with RT, NGS detected more uncommon mutations and improved off-label drugs treatments. The findings highlight the importance of NGS detection, lead to a clinical benefit in uncommon mutations.

\section{Background}

The incidence of lung cancer is increasing worldwide, and non-small cell lung cancer (NSCLC) is one of the main pathological types of lung cancer [1]. NSCLC is classified into two major histological subtypes, adenocarcinoma and squamous-cell carcinoma, and adenocarcinoma accounts for approximately $85 \%$ of lung cancer cases [2]. The emergence of targetable oncogenic driver alterations, most notably epidermal growth factor receptor (EGFR) activating mutations and anaplastic lymphoma kinase (ALK) and ROS1 gene rearrangements, has transformed NSCLC treatment models by incorporating tumor genotyping into therapeutic strategies [3-6]. The response rate of drugs targeting cancers harboring 
driver mutations is $>70 \%$, and these drugs have consistently improved patient survival outcomes. As a result, molecular testing for EGFR, ALK, and ROS1 has been recommended for all adenocarcinoma NSCLC patients since the early days of individualized precision medicine.

Advances in gene detection technology and the rapid development of new drugs have led to the identification of a growing number of driver gene mutations such as ret proto-oncogene (RET) rearrangements, neurotrophic tyrosine receptor kinase 1 (NTRK1), ERBB2 (HER2), and BRAF mutation, MET gene alterations, and other examples of actionable alterations with proven therapeutic options [712]. Companion diagnostic tests for gene mutations that impact the success of anticancer drugs were conducted separately for each mutation, which detected EGFR, ALK, and ROS1 as the main genes. Although the incidence of rare driver gene mutations (mutation rate $<5 \%$ ) such as those of MET, BRAF, NTRK, and HER2 is relatively low and may vary according to ethnic differences, agents targeting these driver mutations are being developed, and the number of emerging biomarkers and targets continues to grow. Targeted therapy is associated with a survival benefit in these patients. Consequently, and increasing number patients undergo multigene sequencing of tumors to identify genomic alterations that can be effectively targeted, and many clinicians are using next generation sequencing (NGS) methods to detect gene mutations associated with lung cancer treatment.

Studies have demonstrated the feasibility of routine NGS detection in patients with NSCLC, and showed that genetic testing using NGS is useful for the design of lung cancer treatment strategies $[14,15]$. However, routine testing may not be sufficient because ethnicity is an important factor determining the response to therapy and outcomes [16]. Presley et al. [17] compared survival outcomes between advanced NSCLC patients who received broad-based genomic sequencing and those who received routine testing for EGFR mutations and/or ALK rearrangements alone in the Community Oncology Setting. They showed that broad-based genomic sequencing provided direct information on treatment in a minority of patients. Singal et al. [18] assessed whether a database with comprehensive genomic profiling could identify and extend associations in NSCLC, and the results demonstrated the feasibility of creating a clinicogenomic database derived from routine clinical experience. Therefore, whether NGS can bring survival benefits to patients compared with routine testing remains controversial. The paucity of appropriate studies and the incomplete understanding of genomic architecture across Chinese populations are additional challenges. In this study, routine testing (EGFR and/or ALK and/or ROS1 only) was compared with NGS panel testing to identify the optimal strategy. We analyzed the difference in the survival benefit between advanced NSCLC patients who used NGS and those undergoing routine testing as well as the potential effect of each test on therapeutic strategies.

\section{Methods}

\section{Study design}

This retrospective cohort study used the Database of Zhejiang Cancer Hospital to identify patients diagnosed with stage IIIB/IV advanced NSCLC who received antineoplastic treatment and either next 
generation sequencing (NGS) or routine testing (EGFR and/or $A L K$ and/or ROS1 only). The histological classification of NSCLC was based on the World Health Organization criteria (2015 version) [19]. Various panels for NGS were used, but all of them included the nine genes used as molecular biomarkers in the National Comprehensive Cancer Network (NCCN) guidelines list of driver mutations (EGFR, ALK, ROS1, KRAS, HER2, BRAF, MET, RET, and NTRK). Propensity score-matched survival analysis was used to evaluate differences in overall survival (OS) between the groups.

\section{Study sample}

Advanced NSCLC patients who were diagnosed between January 1, 2013 and June 30, 2019 and had genetic testing results were included in the study. The cohort included advanced NSCLC patients or earlystage NSCLC that subsequently developed recurrent or progressive disease during the period. Each patient had a diagnosis of lung cancer, at least two documented clinical visits on or after January 1 , 2011, pathology consistent with non-squamous NSCLC, and confirmation of advanced NSCLC on or after January 1, 2011. Patients with evidence of other concurrent active cancers within 6 months prior to diagnosis of advanced NSCLC, other than non-melanoma skin cancer, were excluded. Patients with NGS testing were considered the treatment group, whereas patients with routine testing for EGFR/ALK/ROS1 were considered the control group as standard-of-care group in this study. Patients who met the following inclusion criteria were included in the study: 1) recorded clinicopathological information, including age and sex, and clinical data, such as pathological typing, genetic testing results and treatment type, 2) pathological examination of tumor specimens with proven records of at least one common driver gene test (EGFR or ALK or ROS1), 3) all patients received at least one line of systemic antineoplastic treatment for advanced NSCLC. Patients were excluded if: (1) clinical data including age, gender, and stage were missing, (2) pathologic examination showed NSCLC NOS or small cell lung cancer, (3) follow-up survival results were missing.

\section{Outcomes}

The last follow-up date of the study was June 30, 2020. OS was defined as the time from the start of firstline treatment to the date of death. Analysis was performed using 12-month or 24-month mortality. OS was used as the outcome in the propensity score-matched survival analysis. Secondary outcomes included genetic alterations and treatments received.

\section{Statistical analysis}

The $\chi 2$ test was used for comparison of the distribution of cohort characteristics and treatment types between the NGS group and routine testing group. Propensity score matching was used to address potential confounding due to the significantly different cohort characteristics. Multivariable logistic regression analysis was used to estimate the propensity score based on age, sex, smoking status, stage, year of diagnosis, EGFR or ALK mutation, comorbidity, a single line of treatment, and immunotherapy in line 1 or line 2 vs. line 3 or line 4 of treatment. The quality of data for each variable was considered before their inclusion in propensity score-matched survival analysis. According to the study by Presley et al. [17] 
and to prevent survival bias caused by certain patient characteristics, six covariates were set as exact covariate matches as follows: EGFR or ALK mutation, receipt of only one line of treatment, year of diagnosis, receipt of immunotherapy in line 1 or line 2 of treatment, and receipt of immunotherapy in line

3 or line 4 of treatment. For the variables with missing data (smoking status, and EGFR or ALK mutation), a dummy variable was used because the missing variables were not missing at random. One-to-one matching was performed using a nearest neighbor algorithm with ratio of 1 and a caliper of 0.01 standard deviation. Survival outcomes were estimated and compared by the Kaplan-Meier method (time $0=$ start of first line treatment) and the log-rank test. Median survival and hazard ratio (HR) (log rank) and 95\% confidence interval $(\mathrm{Cl})$ were reported. All statistical tests were two-sided, and a $\mathrm{P}$ value $<0.05$ was considered statistically significant. R version 3.6.0 (R-project, Institute for Statistics and Mathematics: packages Matchlt V3.0.2) was used for all analyses.

\section{Results}

\section{Baseline characteristics}

Of 6,451 patients diagnosed with advanced NSCLC, 85 (12.2\%) received NGS testing (Table 1). The median age at diagnosis was 61 years (range, 19-90 years), $45.2 \%$ of patients had a history of smoking, and $41.6 \%$ of patients were female. In this cohort, $95.8 \%$ of patients received EGFR testing and $95.3 \%$ received ALK testing (in $<5 \%$ of the remaining samples, the testing status of both genes was unknown). There was a significant association between immunotherapy in the first four lines of treatment and NGS testing.

\section{Survival analysis}

All survival analyses were performed using the Kaplan-Meier method. For the entire cohort, the median OS was 22.6 months and the 5 -year survival rate was $22.89 \%$. There was a significant difference in survival between patients who received targeted therapy and those who did not in the unadjusted survival curves (HR, 0.52 [95\% Cl, 0.49-0.56], log-rank $P<0.0001$, Figure 1A) or among the 2177 matched pairs with wellmatched characteristics and balance in the propensity score analysis $(\mathrm{HR}, 0.56$ [95\% Cl, 0.52-0.60], logrank $\mathrm{P}<0.0001$, Fig. 1B).

\section{Survival of patients with NGS testing and routine testing}

OS was compared between patients who underwent NGS testing and those who underwent routine testing. In the unadjusted survival curves, there was an absolute difference between the two cohorts (HR, 0.87 [95\% $\mathrm{Cl}, 0.78-0.96$ ], log-rank $\mathrm{P}=0.0097$, Fig. $2 \mathrm{~A}$ ), especially in the 12- and 24-month mortality rates. In the propensity score analysis, there was no obvious OS difference between the two cohorts (760 matched patients, HR, 0.95 [95\% Cl, 0.82-1.11], log-rank P = 0.53, Fig. 2B). Patients who received NGS had a significantly higher 1 -year survival rate than that of patients with routine testing $(\mathrm{HR}, 0.66[95 \% \mathrm{Cl}$, $0.53-0.83]$, log-rank $P=0.0004)$, as well as a higher rate of patients receiving targeted therapy $(68.0 \% \mathrm{vs}$. 
$53.6 \%, \mathrm{P}<0.0001$, Fig. 3A/B) and patients receiving off-label drugs $(28.10 \%$ vs. $11.40 \%, \mathrm{P}<0.0001$, Fig. $3 C / D)$. The NGS testing cohort had a greater number of different types of off-label drugs.

\section{Survival of patients with uncommon mutations}

In NSCLC, common mutations include EGFR 19del and L858R mutations, and ALK and ROS1 fusion. Here, we defined mutations other than these common mutations as uncommon mutations. In patients with uncommon mutations, OS was compared between patients who received NGS testing and those who underwent routine testing. There was an absolute difference between the two cohorts (HR, 0.79 (95\% $\mathrm{Cl}, 0.69-0.91)$, log-rank $\mathrm{P}=0.0038$, Fig. $4 \mathrm{~A})$ in the unadjusted survival curves, especially in the 12-month mortality ( $\mathrm{HR}, 0.58$ [95\% Cl, 0.48-0.70], log-rank $\mathrm{P}<0.0001)$. After using the propensity score analysis, the 12-month mortality between the two cohorts in 314 matched pairs was significantly different (HR, 0.72 [95\% Cl, 0.54-0.97], log-rank $P=0.03$ ) (Supplementary Fig. 1), although the OS of these patients was not significantly different (HR, 0.86 [95\% $\mathrm{Cl}, 0.69-1.08]$, log-rank $P=0.19$, Fig. 4B).

Next, the patients were divided into four groups: patients with uncommon mutations who received NGS, those who received routine testing, those who received targeted therapies, and those who did not receive targeted therapies. The results of unadjusted survival curves showed that the median OS was longer in the two groups with targeted therapies, especially in the groups with NGS testing (Supplementary Fig. 2). Among uncommon mutation patients with targeted therapies, propensity score analysis showed that the 12- and 24-month mortality was significantly higher in the NGS testing group than in the routine testing group (Supplementary Fig. 2). This suggested that NGS was a better detection method for patients with uncommon mutations. This could be associated with the fact that the rate of off-label treatments was significantly higher in the NGS group than in the routine testing group ( $45.4 \%$ vs. $9.6 \%, P<0.0001$, Supplementary Fig. 3), and indicated that NGS testing resulted in a more objective and precise clinical therapy for uncommon mutation patients.

\section{Treatments received}

All patients received first-line treatment, and 3,517 (54.52\%) and 1,326 (20.56\%) received second- and third-line treatments, respectively. Patients receiving NGS testing were more likely to receive targeted therapy $(51.7 \%)$ as first-line treatment than routine testing patients $(34.6 \%, \mathrm{P}<0.0001)$, whereas the opposite result was obtained for second-line treatment (19.8\% for NGS testing vs. $25.1 \%$ for routine testing, $P=0.012$, Table 2$)$ and for third-line treatment $(12.9 \%$ vs. $15.4 \%, P=0.333)$. The rate of chemotherapy \pm anti-VEGF treatment as first-line treatment was lower in the NGS testing group than in the routine testing group ( $39.5 \%$ vs. $62.5 \%, \mathrm{P}<0.0001)$, whereas it was similar between the two cohorts in the second- and third-line treatments. In the first-, second- and third-line therapy groups, patients receiving NGS testing were more likely to receive immunotherapy (1st-line: $3.6 \%$ vs. $1.0 \%$, 2nd-line: $8.3 \%$ vs. $2.8 \%$, 3rd-line: $4.6 \%$ vs. $1.8 \%$ ) or participate in clinical trials (1st-line: $5.2 \%$ vs. $1.9 \%$, 2 nd-line: $5.7 \%$ vs. $3.0 \%$, 3rdline: $2.5 \%$ vs. $1.7 \%$ ) than those receiving routine testing (Table 2). Among patients without EGFR mutations or ALK fusions, the percentage receiving targeted therapies, immunotherapy, and clinical trials 
was higher in the NGS testing group than in the routine testing group. Correspondingly, the percentage receiving chemotherapy \pm anti-VEGF treatment showed the opposite trend (Table 3 ).

\section{Discussion}

Two different survival analysis methods were used to show that NGS detection technology based on accessible targeted therapy could extend the overall survival of advanced NSCLC patients by nearly 3 months compared with routine detection technology including EGFR and/or ALK and/or ROS1 in the Chinese population. In particular, the 1-year survival rate of patients undergoing NGS testing was significantly higher than that of patients undergoing routine testing. Propensity score analysis confirmed the significant difference in the 1-year survival rate between the two groups. These differences may be attributed to the higher proportion of patients receiving targeted therapy and off-label treatment in the NGS group than in the routine testing group. To the best of our knowledge, this is the first presentation of real world clinical data supporting that NGS is superior for clinical molecular profiling of advanced NSCLC patients, which may improve the survival of patients with advanced NSCLC in the Chinese population. However, we also need to consider the availability of targeted drugs in China.

Genotyping is an essential step for the selection of targeted regimens in lung cancer, especially in lung adenocarcinoma. The survival benefits of targeted therapy are higher than those of chemotherapy in patients with lung cancer. The present results showed that there was a significant difference in survival between patients with and without targeted therapy. The median OS of NSCLC patients treated with targeted therapy was 30 months, and the 5 -year survival rate was $29.6 \%$ in this study. These findings are consistent with the results of previous studies $[14,18]$ and suggested that driver and genotype-directed therapy improved the survival of patients. Comprehensive survival analysis of patients receiving NGS and routine testing was performed to explore the difference between the two techniques in clinical practice. The results showed a difference in OS between patients detected by NGS and routine testing; the OS of NSCLC patients in the NGS group was 2.7 months longer than that of the routine detection group $(\mathrm{P}=$ 0.0097). In particular, the 1-year survival rate was significantly higher in the NGS group than in the routine testing group ( $82.03 \%$ vs. $70.76 \%, P<0.0001)$. This may be related to the obviously higher rate of patients who received targeted therapy. However, propensity score analysis showed no difference in OS between the two groups, and the 5-year survival did not differ significantly, which was consistent with previous data [14]. The use of NGS in advanced NSCLC patients is already fully endorsed in many international guidelines. According to previous reports, the value of routine testing and NGS testing is still controversial. Singal et al. [18] demonstrated the feasibility of creating a comprehensive genomic profiling derived from routine clinical experience and the use of large-scale clinicogenomic data sets can augment various stages of drug development. Presley et al. [17] demonstrated that broad-based genomic sequencing was not associated with better survival compared with routine testing among advanced NSCLC patients receiving treatment. Similar results were obtained in the present study, which showed no obvious OS difference using propensity score analysis $(P=0.53)$. This may indicate that the overall survival benefit of NGS in lung adenocarcinoma patients is not large, and a specific population is necessary to regulate the detection by NGS. 
NGS may increase the opportunities for targeted therapies. Wu et al. [20] described the Pan-Asian guidelines established by ESMO and the Chinese Society of Clinical Oncology recommending testing of all advanced non-squamous NSCLC patients for EGFR mutation, ALK rearrangement, ROS1

rearrangement, and BRAF mutation. The IALSC Updated Molecular Testing Guidelines recommend testing for EGFR, ALK, and ROS1 along with the inclusion of additional genes (ERBB2, MET, BRAF, KRAS, and RET) for NGS panels [7]. These findings suggest that NGS testing is becoming increasingly important for confirming the genetic features of patients, and effective targeted drugs may be needed to demonstrate the improved outcomes of NSCLC patients using NGS. However, only three categories of targeted drugs (EGFR, ALK, and ROS1) have been approved in China, and access to other drugs is limited. In this study, NGS testing was not superior for guiding targeted therapy in second- and third-line treatments, which may be due to the limited targeted drugs against EGFR mutations or ALK fusions that can be easily checked by routing testing. NGS is used in second- and third-line treatments to explore drug resistance mechanisms, as the corresponding targeted drugs are limited. Therefore, the proportion of off-label patients increased after NGS testing in China. In addition, the 36-month mortality and OS did not differ significantly between the NGS testing and routine testing groups, suggesting that not all targeted drugs offered an OS benefit. Because off-label medication is usually selected after the failure of standard treatment, the patient benefit from off-label drugs may be lower than that of first line treatment.

NGS testing can identify specific mutations that cannot be found by routine testing. In the present study, the OS of uncommon mutation patients and that of patients receiving targeted therapies with NGS testing was significantly longer than that of patients with routine testing. On the other hand, the rate of off-label treatments was significantly higher in the NGS than in the routine testing group (45.4\% vs. 9.6\%; $P<0.0001$ ), suggesting that NGS testing resulted in a more objective and precise clinical therapy for patients with uncommon mutations. In addition, NGS may help patients with specific mutations participate in clinical trials ( $9.6 \%$ vs. $3.9 \%)$. Zehir et al. [13] established a large-scale, prospective clinical sequencing initiative using a comprehensive assay (MSK-IMPACT trial), and found that only $11 \%$ of patients were enrolled in genomic-matched clinical trials among 10,000 patients. In the present study, the proportion of patients matched to clinical trials based on NGS was $9.6 \%$, which was slightly lower than that of the previous study. This may be because clinical trials in China were somewhat similar to foreign community models. The study of Presley et al. [17] focused mainly on community-based practice, and demonstrated that the number of patients treated in the community setting who have access to clinical trials for advanced NSCLC remained low. A previous study [21] explored changes in clinical trials of cancer drugs in China over the decade of 2009-2018, and the results showed an increase in the number of clinical trials over time. The proportion of Phase 1 trials being initiated increased as well. However, the number of clinical trials focusing on epidemiological characteristics unique to the Chinese population remains low, and the geographical distribution of leading clinical trial units is unbalanced. Therefore, increased access to research clinical trials in China may improve the use of mutational data by NGS testing. The crossover of survival analysis curves of NGS and routine testing may be due to the fact that NGS panels derived from routine clinical practice may be mixed, and some patients cannot afford 
expensive targeted drugs, or the conditions were not met for inclusion in clinical studies. Overall, although the benefits of NGS for NSCLC patients are limited, NGS remains essential in clinical practice.

The present study had several limitations, which need to be considered for data interpretation. First, this was a retrospective study, which may affect the results. For example, patient heterogeneity, inconsistency of treatment options selected by clinicians, and the fact that not all patients received NGS detection at diagnosis were factors that need to be considered. Second, this was not a randomly assigned study and this may induce analysis bias. Third, other outcome points including overall response rate and progression-free survival were not analyzed in this study. Fourth, despite the propensity analysis, confounding factors were not fully excluded, and unknown confounding factors may have been included. For patients undergoing NGS testing, the NGS panel contained the gene mutations mentioned in the NCCN guidelines; however, the panels were derived from different testing platforms and were not all the same. We considered that our study still had some clinical significance. Our article conducted out a large sample to confirm the NGS value compared by routine testing and it also suggest that NGS may need to be used more accurately. Future studies should assess the value of NGS in clinical trials of new drugs and the benefits of NGS in clinical practice after the approval of new drugs.

\section{Conclusions}

NGS technology allowed the detection of uncommon driver mutations, which may lead to a greater benefit from targeted treatments and participation in clinical trials in NSCLC; however, it was not independently associated with a large survival benefit in advanced NSCLC patients. The patient populations need to be further categorized to explore the value of NGS in clinical practice.

\section{Abbreviations}

NGS: next generation sequencing, NSCLC: non-small cell lung cancer, OS: Overall survival, EGFR: epidermal growth factor receptor, ALK: anaplastic lymphoma kinase, RET: ret proto-oncogene, NTRK1: neurotrophic tyrosine receptor kinase 1, NCCN: National Comprehensive Cancer Network, HR: hazard ratio.

\section{Declarations}

\section{Ethics approval and consent to participate}

This study was conducted in accordance with the Declaration of Helsinki. The protocol was approved by the Institutional Ethics Committee at Zhejiang Cancer Hospital.

\section{Consent for publication}

All authors read this manuscript and approve for publication. 


\section{Competing interests}

Xiaoyan Zhang and Tonghui Ma are the employees of Genetron Health (Beijing) Technology, Co. Ltd. All other authors declare no conflict of interests.

\section{Funding}

This study was funded by the Zhejiang Chinese Medical Science and Technology Foundation (No.2021ZQ013) and Xisike-Hanson Cancer Research Foundation (Y-HS2019-20).

\section{Authors' contributions}

SZB, ZYP funded this study, WWX, SZB, ZXY designed project and wrote the

manuscript. SJF, LGY, SZY, SL collected samples and clinical data. WWX, ZXY, MTH performed data analysis, SZB, ZYP revised the manuscript. All authors read and approved the final manuscript.

\section{Acknowledgements}

Not applicable.

\section{Availability of data and materials}

The datasets used during the current study are available from the corresponding author on reasonable request.

\section{References}

1. Siegel RL, Miller KD, Jemal A. Cancer statistics, 2020. CA Cancer J Clin. 2020,70(1):7-30. doi:10.3322/caac.21590.

2. Bade BC, Dela Cruz CS. Lung Cancer 2020: Epidemiology, etiology, and prevention. Clin Chest Med. 2020,41(1):1-24. doi:10.1016/j.ccm.2019.10.001.

3. Mitsudomi T, Morita S, Yatabe Y, Negoro S, Okamoto I, Tsurutani J, Seto T, Satouchi M, Tada H, Hirashima T, et al. Gefitinib versus cisplatin plus docetaxel in patients with non-small-cell lung cancer harbouring mutations of the epidermal growth factor receptor (WJTOG3405): an open label, randomised phase 3 trial. Lancet Oncol. 2010,11(2):121-8. doi: 10.1016/S1470-2045(09)70364-X.

4. Shaw AT, Ou SH, Bang YJ, Camidge DR, Solomon BJ, Salgia R, Riely GJ, Varella-Garcia M, Shapiro GI, Costa DB, et al. Crizotinib in ROS1-rearranged non-small-cell lung cancer. N Engl J Med. 2014,371(21):1963-71. doi: 10.1056/NEJMoa1406766.

5. Shaw AT, Kim DW, Nakagawa K, Seto T, Crinó L, Ahn MJ, De Pas T, Besse B, Solomon BJ, Blackhall F, et al. Crizotinib versus chemotherapy in advanced ALK-positive lung cancer. $\mathrm{N}$ Engl J Med. 2013,368(25):2385-94. doi: 10.1056/NEJMoa1214886. 
6. Peters S, Camidge DR, Shaw AT, Gadgeel S, Ahn JS, Kim DW, Ou SI, Pérol M, Dziadziuszko R, Rosell R, et al. Alectinib versus Crizotinib in Untreated ALK-Positive Non-Small-Cell Lung Cancer. N Engl J Med. 2017,377(9):829-838. doi: 10.1056/NEJMoa1704795.

7. Lindeman NI, Cagle PT, Aisner DL, Arcila ME, Beasley MB, Bernicker EH, Colasacco C, Dacic S, Hirsch FR, Kerr K, et al. Updated Molecular Testing Guideline for the Selection of Lung Cancer Patients for Treatment With Targeted Tyrosine Kinase Inhibitors: Guideline From the College of American Pathologists, the International Association for the Study of Lung Cancer, and the Association for Molecular Pathology. J Thorac Oncol. 2018,13(3):323-358. doi: 10.1016/j.jtho.2017.12.001.

8. Salama AKS, Li S, Macrae ER, Park JI, Mitchell EP, Zwiebel JA, Chen HX, Gray RJ, McShane LM, Rubinstein LV, et al. Dabrafenib and Trametinib in Patients With Tumors With BRAFV600E Mutations: Results of the NCI-MATCH Trial Subprotocol H. J Clin Oncol. 2020,38(33):3895-3904. doi: $10.1200 / J C O .20 .00762$.

9. Koga T, Kobayashi Y, Tomizawa K, Suda K, Kosaka T, Sesumi Y, Fujino T, Nishino M, Ohara S, Chiba $M$, et al. Activity of a novel HER2 inhibitor, poziotinib, for HER2 exon 20 mutations in lung cancer and mechanism of acquired resistance: An in vitro study. Lung Cancer. 2018,126:72-79. doi: 10.1016/j.lungcan.2018.10.019.

10. Farago AF, Le LP, Zheng Z, Muzikansky A, Drilon A, Patel M, Bauer TM, Liu SV, Ou SH, Jackman D, et al. Durable Clinical Response to Entrectinib in NTRK1-Rearranged Non-Small Cell Lung Cancer. J Thorac Oncol,10(12):1670-4. doi: 10.1097/01.JT0.0000473485.38553.f0.

11. Yang H, Zhou Z, Lin L, Yang M, Li C, Li Z, Yu X, Lizaso A, Han-Zhang H, Li B, et al. Characterization of MET exon 14 alteration and association with clinical outcomes of crizotinib in Chinese lung cancers. Lung Cancer. 2020,148:113-121. doi: 10.1016/j.lungcan.2020.08.009.

12. Passiglia F, Malapelle U, Del Re M, Righi L, Pagni F, Furlan D, Danesi R, Troncone G, Novello S. KRAS inhibition in non-small cell lung cancer: Past failures, new findings and upcoming challenges. Eur $\mathrm{J}$ Cancer. 2020,137:57-68. doi: 10.1016/j.ejca.2020.06.023.

13. Zehir A, Benayed R, Shah RH, Syed A, Middha S, Kim HR, Srinivasan P, Gao J, Chakravarty D, Devlin $\mathrm{SM}$, et al. Mutational landscape of metastatic cancer revealed from prospective clinical sequencing of 10,000 patients. Nat Med. 2017,23(6):703-713. doi: 10.1038/nm.4333.

14. Kris MG, Johnson BE, Berry LD, Kwiatkowski DJ, lafrate AJ, Wistuba II, Varella-Garcia M, Franklin WA, Aronson SL, Su PF, et al. Using multiplexed assays of oncogenic drivers in lung cancers to select targeted drugs. JAMA. 2014,311(19):1998-2006. doi: 10.1001/jama.2014.3741.

15. Yamamoto G, Kikuchi M, Kobayashi S, Arai Y, Fujiyoshi K, Wakatsuki T, Kakuta M, Yamane Y, lijima Y, Mizutani $\mathrm{H}$, et al. Routine genetic testing of lung cancer specimens derived from surgery, bronchoscopy and fluid aspiration by next generation sequencing. Int J Oncol. 2017,50(5):15791589. doi: 10.3892/ijo.2017.3935.

16. Tan DS, Mok TS, Rebbeck TR. Cancer Genomics: Diversity and Disparity Across Ethnicity and Geography. J Clin Oncol. 2016,34(1):91-101. doi: 10.1200/JC0.2015.62.0096. 
17. Presley CJ, Tang D, Soulos PR, Chiang AC, Longtine JA, Adelson KB, Herbst RS, Zhu W, Nussbaum NC, Sorg RA, et al. Association of Broad-Based Genomic Sequencing With Survival Among Patients With Advanced Non-Small Cell Lung Cancer in the Community Oncology Setting. JAMA. 2018,320(5):469-477. doi: 10.1001/jama.2018.9824.

18. Singal G, Miller PG, Agarwala V, Li G, Kaushik G, Backenroth D, Gossai A, Frampton GM, Torres AZ, Lehnert EM, et al. Association of Patient Characteristics and Tumor Genomics With Clinical Outcomes Among Patients With Non-Small Cell Lung Cancer Using a Clinicogenomic Database. JAMA. 2019,321(14):1391-1399. doi: 10.1001/jama.2019.3241.

19. Travis WD, Brambilla E, Burke AP, Marx A, Nicholson AG. WHO Classification of tumours of the lung, pleura, thymus and heart. 4th ed. Lyon: International Agency for Research on Cancer. 2015: 9-96.

20. Wu YL, Planchard D, Lu S, Sun H, Yamamoto N, Kim DW, Tan DSW, Yang JC, Azrif M, Mitsudomi T,et al. Pan-Asian adapted Clinical Practice Guidelines for the management of patients with metastatic non-small-cell lung cancer: a CSCO-ESMO initiative endorsed by JSMO, KSMO, MOS, SSO and TOS. Ann Oncol. 2019,30(2):171-210. doi: 10.1093/annonc/mdy554.

21. Li N, Huang HY, Wu DW, Yang ZM, Wang J, Wang JS, Wang SH, Fang H, Yu Y, Bai Y, et al. Changes in clinical trials of cancer drugs in mainland China over the decade 2009-18: a systematic review. Lancet Oncol. 2019,20(11):e619-e626. doi: 10.1016/S1470-2045(19)30491-7.

\section{Tables}

Table 1 Characteristics of the sample of patients with advanced non-small cell lung cancer $(N=6451)$ 


\begin{tabular}{|c|c|c|c|}
\hline \multirow[b]{2}{*}{ Total } & \multicolumn{2}{|l|}{ No. (\%) } & \multirow[t]{2}{*}{ P Value } \\
\hline & NGS $(n=785)$ & Routine Testing ( $n=5666)$ & \\
\hline \multicolumn{4}{|l|}{ Age at diagnosis, y } \\
\hline$\leq 45$ & $68(8.7)$ & $388 \rrbracket 6.8 \rrbracket$ & \multirow{5}{*}{0.007} \\
\hline $46-55$ & $215(27.4)$ & $1330 \rrbracket 23.5 \rrbracket$ & \\
\hline $56-65$ & 308(39.2) & 2249ه39.7ه & \\
\hline $66-75$ & $165(21.0)$ & 1450ه25.6ه & \\
\hline$\geq 76$ & $29(3.7)$ & $249 \rrbracket 4.4 \rrbracket$ & \\
\hline \multicolumn{4}{|l|}{ Sex } \\
\hline Male & $433(55.2)$ & $3334 \llbracket 58.8 \rrbracket$ & \multirow[b]{2}{*}{0.050} \\
\hline Female & $352(44.8)$ & $2332 \varangle 41.2 \rrbracket$ & \\
\hline \multicolumn{4}{|l|}{ Comorbidity count ${ }^{\mathrm{b}}$} \\
\hline 0 & $513(65.4)$ & $2794 \llbracket 49.3 \rrbracket$ & \multirow{3}{*}{$<0.0001$} \\
\hline $1-2$ & 266(33.8) & $2660 \rrbracket 47.0 \rrbracket$ & \\
\hline$\geq 3$ & $6(0.8)$ & $212 \rrbracket 3.7 \rrbracket$ & \\
\hline \multicolumn{4}{|l|}{ Smoking status ${ }^{c}$} \\
\hline No history of smoking & $435 \llbracket 55.4 \rrbracket$ & $2896 \rrbracket 51.1 \rrbracket$ & \multirow{3}{*}{$<0.0001$} \\
\hline History of smoking & $346 \rrbracket 44.1 \rrbracket$ & $2574 \llbracket 45.4 \rrbracket$ & \\
\hline Unknown & $4 \rrbracket 0.5 \rrbracket$ & $196 \rrbracket 3.5 \rrbracket$ & \\
\hline \multicolumn{4}{|l|}{ Medical payment method } \\
\hline city & $358 \rrbracket 45.6 \rrbracket$ & $2474 \llbracket 43.6 \rrbracket$ & \multirow{3}{*}{0.001} \\
\hline country & $115 \rrbracket 14.6 \rrbracket$ & $1185 \llbracket 20.9 \rrbracket$ & \\
\hline self-paying & $2 \varangle 0.3 \rrbracket$ & $9 \rrbracket 0.2 \rrbracket$ & \\
\hline Unknown & $310 \rrbracket 39.5 \rrbracket$ & 1998囚35.3囚 & \\
\hline \multicolumn{4}{|c|}{ Income, by medical payment method } \\
\hline 1 (lowest) & 117ه14.9凶 & $1194 \llbracket 21.0 \rrbracket$ & \multirow[t]{2}{*}{$<0.0001$} \\
\hline 2(highest) & $358 \rrbracket 45.6 \rrbracket$ & $2474 \llbracket 43.7 \rrbracket$ & \\
\hline Unknown & $310 \varangle 39.5 \rrbracket$ & 1998凶35.3囚 & \\
\hline
\end{tabular}




\begin{tabular}{|c|c|c|c|}
\hline I & 15ه1.9凶 & 208ه3.7》 & \multirow{4}{*}{$<0.0001$} \\
\hline II & $15 \llbracket 1.9 \rrbracket$ & $214 \rrbracket 3.8 \rrbracket$ & \\
\hline III & $93 \otimes 11.8 \rrbracket$ & 1071ه18.9凶 & \\
\hline IV & $662 \rrbracket 84.4 \rrbracket$ & $4173 \otimes 73.6 \rrbracket$ & \\
\hline \multicolumn{4}{|l|}{ Year of diagnosis } \\
\hline 2013 & $0 \otimes 0 \bigotimes$ & $588 \otimes 10.4 \rrbracket$ & \multirow{7}{*}{$<0.0001$} \\
\hline 2014 & 13凶1.7凶 & $785 \rrbracket 13.9 \rrbracket$ & \\
\hline 2015 & $29 \bowtie 3.7 \rrbracket$ & $987 \rrbracket 17.4 \rrbracket$ & \\
\hline 2016 & 156冈19.9凶 & 1062ه18.7区 & \\
\hline 2017 & $224 \llbracket 28.5 \rrbracket$ & 1051ه18.6ه & \\
\hline 2018 & $259 \llbracket 33.0 \rrbracket$ & $920 \rrbracket 16.2 \rrbracket$ & \\
\hline 2019 & 104ه13.2】 & $273 \rrbracket 4.8 \rrbracket$ & \\
\hline \multicolumn{4}{|l|}{$E G F R$ status $^{d}$} \\
\hline Total tested & $785 \llbracket 100.0 \rrbracket$ & $5666 \rrbracket 100.0 \rrbracket$ & \\
\hline Positive & $415 \llbracket 52.9 \rrbracket$ & $2449 \llbracket 43.2 \rrbracket$ & \multirow[t]{2}{*}{$<0.0001$} \\
\hline Negative & $370 \rrbracket 47.1 \rrbracket$ & $2946 \rrbracket 52.0 \rrbracket$ & \\
\hline Unknown & & $0 \otimes 0.0 \rrbracket$ & $271 \rrbracket 4.8 \rrbracket$ \\
\hline \multicolumn{4}{|l|}{ ALK status } \\
\hline Total tested & $785 \rrbracket 100 \rrbracket$ & $5666 \rrbracket 100 \rrbracket$ & \\
\hline Positive & $40 \rrbracket 5.1 \rrbracket$ & $294 \rrbracket 5.2 \rrbracket$ & \multirow[t]{2}{*}{$<0.0001$} \\
\hline Negative & $740 \rrbracket 94.3 \rrbracket$ & $5073 \rrbracket 89.5 \rrbracket$ & \\
\hline Unknown & $5 \rrbracket 0.6 \rrbracket$ & $299 \rrbracket 5.3 \rrbracket$ & \\
\hline \multicolumn{4}{|c|}{ Timing of next generation sequencing } \\
\hline Before first-line treatment & $522 \bowtie 66.5 \rrbracket$ & & \\
\hline Before second-line treatment & $165 \rrbracket 21.0 \rrbracket$ & & \\
\hline Before third-line treatment & $51 \rrbracket 6.5 \rrbracket$ & & \\
\hline Before fourth-line treatment & $20 \bigotimes 2.5 \rrbracket$ & & \\
\hline Before fifth-line treatment & $3 \llbracket 0.4 \rrbracket$ & & \\
\hline Before sixth-line treatment & $2 \bowtie 0.3 \rrbracket$ & & \\
\hline
\end{tabular}


Table 2 Treatments received by patients with advanced non-small cell lung cancer. 


\begin{tabular}{|c|c|c|c|}
\hline \multirow{3}{*}{ Treatment Type } & \multicolumn{3}{|l|}{ No. (\%) } \\
\hline & NGS & Routine Testing & \\
\hline & $(n=785)$ & $(n=5666)$ & $P$ Value \\
\hline \multicolumn{4}{|l|}{ First-line treatment } \\
\hline Targeted treatment & $406(51.7)$ & 1961(34.6) & $<0.0001$ \\
\hline Chemotherapy \pm anti-VEGF & $310(39.5)$ & $3541(62.5)$ & $<0.0001$ \\
\hline Immunotherapy & $28(3.6)$ & $55(1.0)$ & $<0.0001$ \\
\hline Clinical trial & $41(5.2)$ & 109ه1.9凶 & $<0.0001$ \\
\hline \multicolumn{4}{|l|}{ Second-line treatment } \\
\hline Targeted treatment & $98(19.8)$ & $758(25.1)$ & 0.012 \\
\hline Chemotherapy \pm anti-VEGF & $327(66.2)$ & 2088(69.1) & 0.201 \\
\hline Immunotherapy & $41(8.3)$ & $84(2.8)$ & $<0.0001$ \\
\hline Clinical trial & $28(5.7)$ & $93(3.0)$ & 0.003 \\
\hline \multicolumn{4}{|l|}{ Third-line treatment } \\
\hline Targeted treatment & $31(12.9)$ & 167(15.4) & 0.333 \\
\hline Chemotherapy \pm anti-VEGF & 192(80.0) & $881(81.1)$ & 0.689 \\
\hline Immunotherapy & $11(4.6)$ & $20(1.8)$ & 0.011 \\
\hline Clinical trial & $6(2.5)$ & 18(1.7) & 0.376 \\
\hline \multicolumn{4}{|l|}{ Fourth-line treatment } \\
\hline Targeted treatment & $31(12.9)$ & $167(15.4)$ & 0.333 \\
\hline Chemotherapy \pm anti-VEGF & 192(80.0) & $881(81.1)$ & 0.689 \\
\hline Immunotherapy & $11(4.6)$ & $20(1.8)$ & 0.011 \\
\hline Clinical trial & $6(2.5)$ & 18(1.7) & 0.376 \\
\hline Total NGS & $785(100)$ & & \\
\hline No NGS-informed targeted treatment & $251(31.9 \%)$ & & \\
\hline Other targeted treatment & $40(5.0 \%)$ & & \\
\hline EGFR/ALK approved targeted treatment & $382(48.7 \%)$ & & \\
\hline$E G F R / A L K$ off-label treatment & $112(14.3 \%)$ & & \\
\hline
\end{tabular}


Table 3 First and second line treatment type received among patients without EGFR or ALK alterations

\begin{tabular}{|llllll|}
\hline Treatment Type & \multicolumn{5}{c|}{ Routine testing } \\
& NGS & & & P-value \\
\hline & $\mathbf{n}$ & $\%$ & $\mathbf{n}$ & $\%$ & \\
\hline First Line Treatment & & & & & \\
\hline Targeted Treatment & 42 & 12.9 & 201 & 8.5 & 0.009 \\
\hline Chemo +/- Anti-VEGF & 230 & 70.7 & 2057 & 87.1 & $<0.0001$ \\
\hline Immunotherapy & 25 & 7.8 & 45 & 1.9 & $<0.0001$ \\
\hline Clinical Trial & 28 & 8.6 & 60 & 2.5 & $<0.0001$ \\
\hline Second Line Treatment & & & & & \\
\hline Targeted Treatment & 55 & 27.2 & 224 & 20.1 & 0.022 \\
\hline Chemo +/- Anti-VEGF & 102 & 50.5 & 793 & 71.1 & $<0.0001$ \\
\hline Immunotherapy & 33 & 16.4 & 62 & 5.6 & $<0.0001$ \\
\hline Clinical Trial & 12 & 5.9 & 36 & 3.2 & 0.058 \\
\hline
\end{tabular}

Figures

A

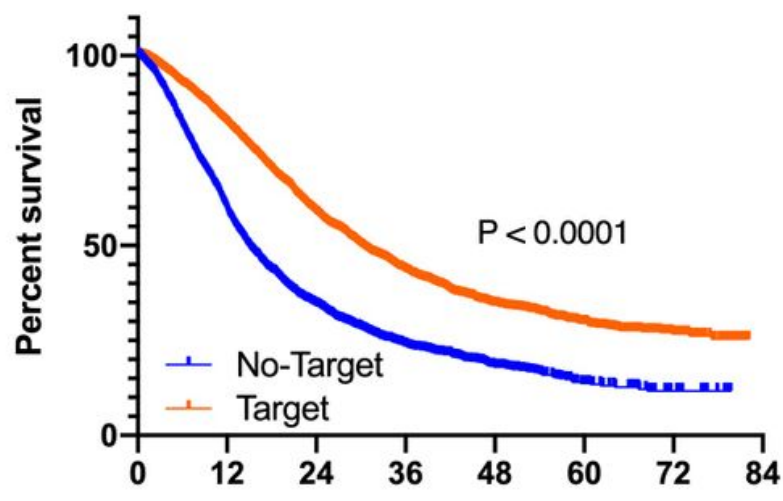

No.at risk

$\begin{array}{rrrrrrrrr}\text { No-target } & 2879 & 1483 & 651 & 322 & 137 & 48 & 15 & 1 \\ \text { Target } & 3571 & 2666 & 1528 & 842 & 437 & 206 & 69 & 1\end{array}$

B

(matched)Target vs. no-target

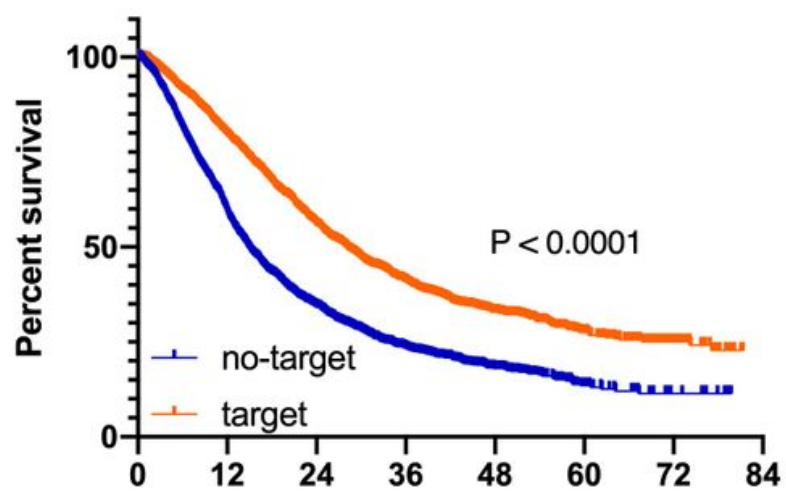

No.at risk

Months

$\begin{array}{rrrrrrrrr}\text { No-target } & 2176 & 1141 & 534 & 262 & 111 & 36 & 12 & 1 \\ \text { Target } & 2177 & 1555 & 872 & 473 & 246 & 108 & 34 & 1\end{array}$ 
Figure 1

Kaplan Meier estimates of overall survival (OS) according to targeted therapy (TT) in all patients and used for a propensity score-matched survival analysis (A) OS between patients with or without TT (30 months vs 14.9 months, $\mathrm{P} \otimes 0.0001)$; (B) matched OS between patients with or without TT (27.9 months vs 14.8 months, $\mathrm{P} \otimes 0.0001)$.

A

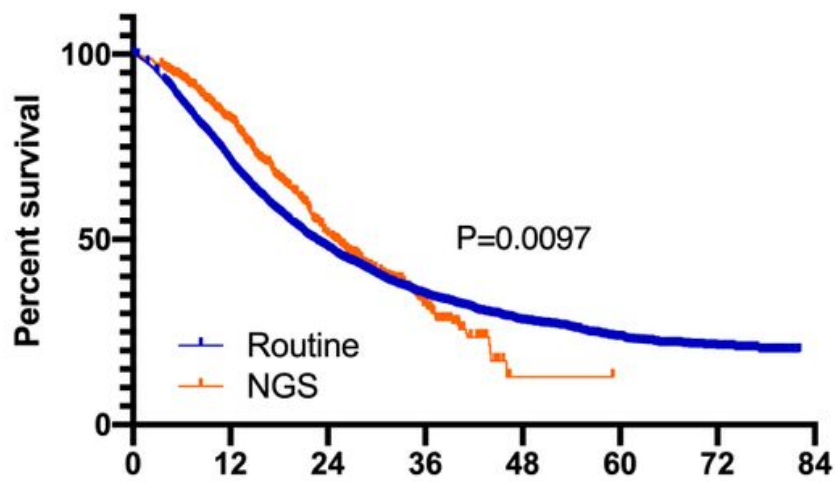

No.at risk

Routine

56
Survival of NGS vs. Routine

\section{Months}

$3643 \quad 1976$

$506 \quad 203$
1104

61
573

$253 \quad 83$ $\begin{array}{lll}1 & 1 & 1\end{array}$
B

(matched) NGS vs. Routine

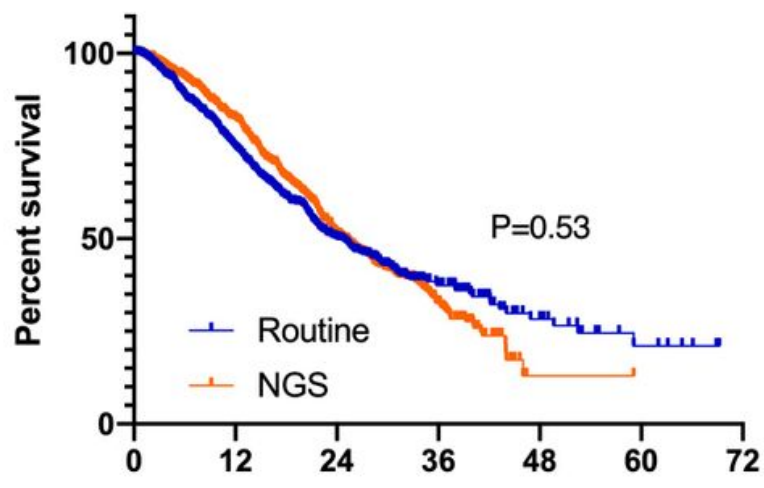

No.at risk

$\begin{array}{cccccccc}\text { Routine } & 760 & 430 & 166 & 65 & 19 & 7 & 1 \\ \text { NGS } & 760 & 500 & 202 & 61 & 2 & 1 & 1\end{array}$

\section{Figure 2}

Kaplan Meier estimates of overall survival (OS) according to NGS or Routine testing in all patients and used for a propensity score-matched survival analysis (A) OS between patients with NGS or with Routine testing (24.8 months vs 22.1 months, $P=0.0097)$; $(B)$ matched OS between patients with NGS or with Routine testing ( 24.8 months vs 24 months, $P=0.53$ ). 


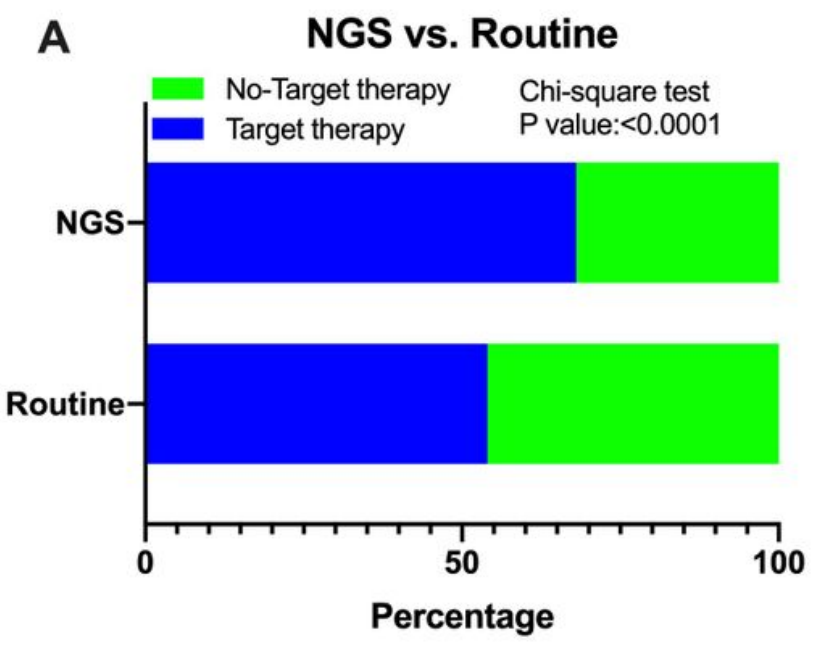

C

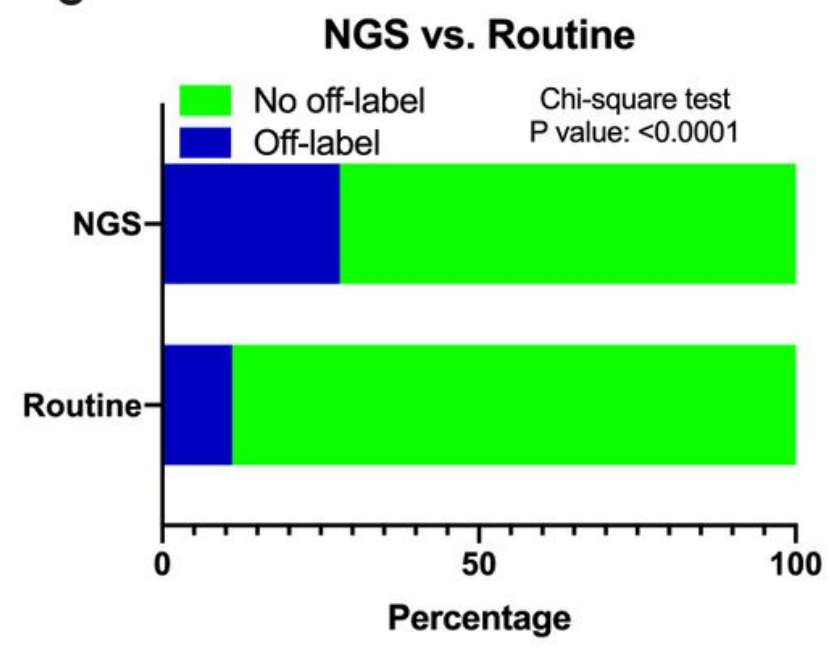

B

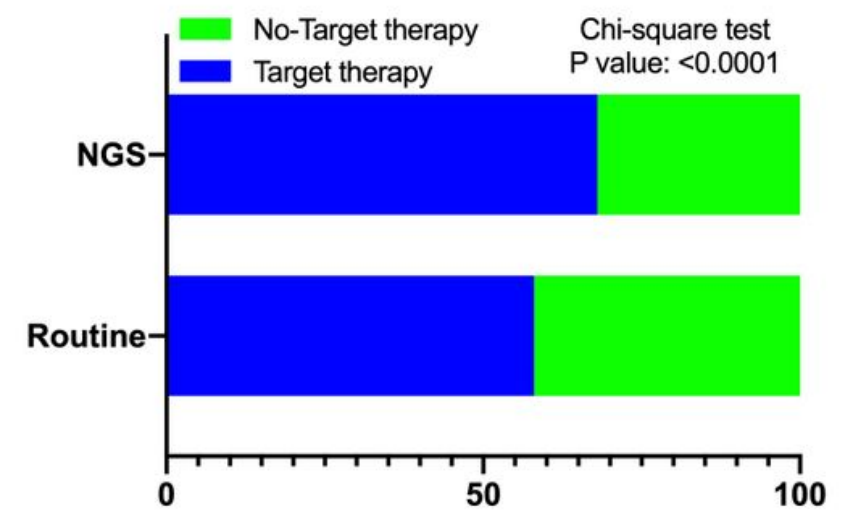

D

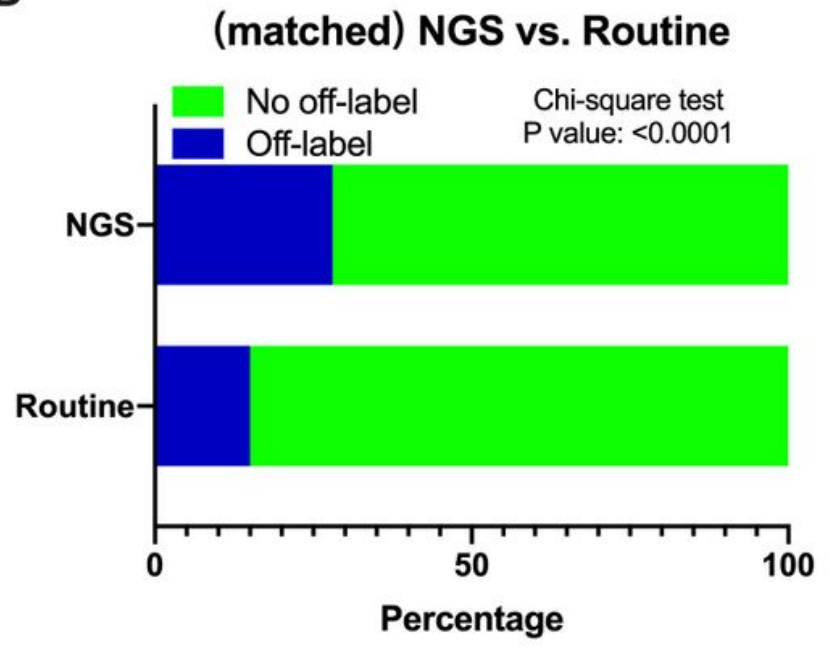

\section{Figure 3}

The comparison of the ration of the patients that received targeted therapy and off-label treatment according to NGS or Routine testing and used for a propensity score-matched survival analysis. The obviously higher ratio of the patients that received targeted therapy $(68.0 \%$ vs. $53.6 \% ; \mathrm{P}<0.0001)$ and that received off-label drugs $(28.10 \%$ vs. $11.40 \%$; $P<0.0001)$. 
A

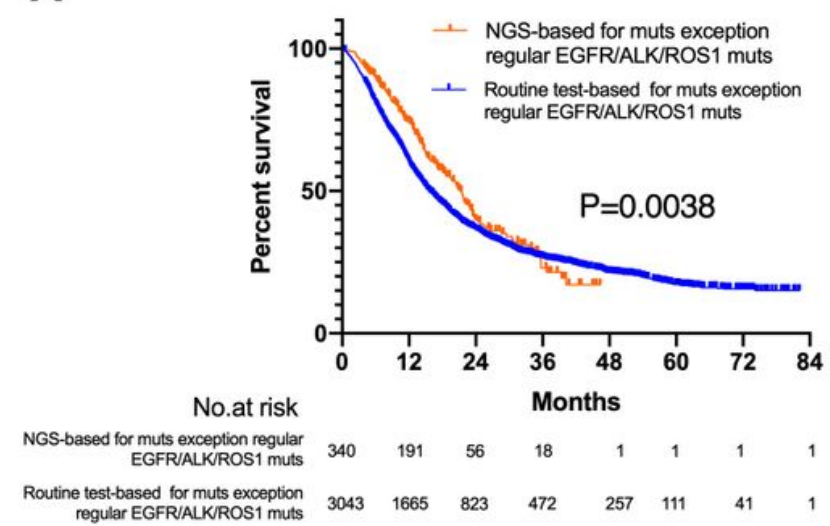

B

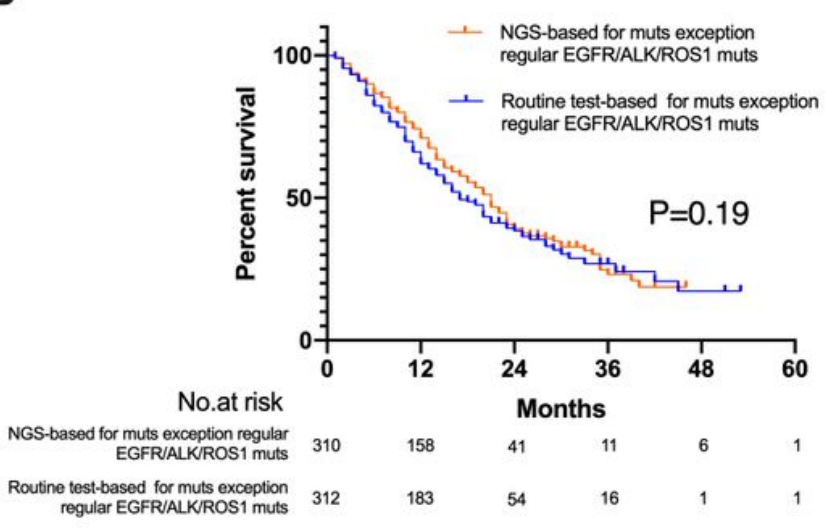

Figure 4

Kaplan Meier estimates of overall survival (OS) according to NGS or Routine testing in uncommon mutated patients and used for a propensity score-matched survival analysis (A) OS between patients with NGS or with Routine testing (21.2 months vs 15.7 months, $P=0.0038$ ); (B) matched OS between patients with NGS or with Routine testing (21 months vs 17 months, $P=0.19$ ).

\section{Supplementary Files}

This is a list of supplementary files associated with this preprint. Click to download.

- NGSSFigure.docx 